\section{AB0811 DIRECT COST OF BIOLOGICS IN RHEUMATOID ARTHRITIS PATIENTS IN A DEVELOPING COUNTRY: RESULTS FOR THE MOROCCAN RBSMR REGISTRY}

S. Farih ${ }^{1}$, H. Rkain ${ }^{1,2}$, S. Fellous ${ }^{1}$, S. Ahid ${ }^{3}$, R. Abouqal ${ }^{4}$, T. Latifa $^{1}$ I. Hmamouchi ${ }^{5,6}$, L. Achemlal ${ }^{7}$, I. El Bouchti ${ }^{8}$, A. EL Maghraoui ${ }^{9}$, I. Ghozlani ${ }^{10}$, H. Hassikou ${ }^{11}$, T. Harzy ${ }^{12}$, I. Linda ${ }^{13}$, O. Mkinsi ${ }^{14}$, R. Niamane ${ }^{15}$, R. Bahiri ${ }^{16}$ F. Allalii. ${ }^{1}$ El Ayachi Hospital, Ibn Sina University Hospital, Department of Rheumatology B, Salé, Morocco; ${ }^{2}$ Faculty of Medicine and Pharmacy of Rabat, Mohammed V University, Physiology Laboratory, Rabat, Morocco; ${ }^{3}$ Mohammed V University, Research Team of Pharmacoeconomics \& Pharmacoepidemiology, Rabat, Morocco; ${ }^{4}$ Faculty of Medicine and Pharmacy, Mohammed V University, Laboratory of Biostatistical, Clinical and Epidemiological Research, Faculty of Medicine and Pharmacy, Rabat, Morocco; ${ }^{5}$ Faculty of Medicine and Pharmacy, Mohammed V University, Laboratory of Biostatistical, Clinical and Epidemiological Research, Rabat, Morocco; ${ }^{6}$ Provincial Hospital of Temara, Department of Rheumatology, Temara, Morocco; ${ }^{7}$. Military Hospital Mohammed V, Ibn Sina University Hospital, Department of Rheumatology, Rabat, Morocco; ${ }^{8}$ Arrazi University Hospital, Department of Rheumatology, Marrakech, Morocco; ${ }^{9}$ Private Medical Office, Private Medical Office, Rabat, Morocco; ${ }^{10}$ University Hospital of Agadir, Department of Rheumatology, Agadir, Morocco; ${ }^{11}$ Military Hospital Moulay Ismail, Hassan II University Hospital, Department of Rheumatology, Meknès, Morocco; ${ }^{12}$ Hassan II University Hospital, Department of Rheumatology, fes, Morocco; ${ }^{13}$ Mohammed VI University Hospital, Department of Rheumatology, Oujda, Morocco; ${ }^{14} \mathrm{Ibn}$ Rochd University Hospital, Department of Rheumatology, Casablanca, Morocco; ${ }^{15}$ Military Hospital Avicenne, Mohammed VI University Hospital, Department of Rheumatology, Marrakech, Morocco; ${ }^{16}$ El Ayachi Hospital, Ibn Sina University Hospital, Department of Rheumatology A, Salé, Morocco

Objectives: The aim of this study was to calculate direct costs amount in rheumatoid arthritis (R.A) patients in the Moroccan register of biologicals RBSMR registry.

Methods: Patients in the Moroccan register of biologicals RBSMR registry with available 1-year data were included. Variables related to socio-economic status, disease and biological therapy were collected. Direct costs included prices of biologics, costs of infusions, and subcutaneous injections. Biologic use and costs were compared.

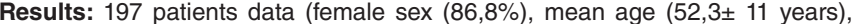
illiteraty $(45,7 \%))$ were analyzed. The median annual direct costs per patient were $€ 1,665$ [€1,472 - €9,879]. For all patients, the mean annual direct costs per treated patient for each biological agent is presented in Figure 1.

The total annual direct costs of biological agents in the RBSMR registry were $978494 €$. Rituximab was given to $67 \%$ of patients and constituted $25.7 \%$ of the total annual budget. TNF-blockers and Tocilizumab were prescribed in $14.2 \%$ and $18.8 \%$ of cases and represented respectively $27.3 \%$ and $47 \%$ of this overall budget.

Figure 1 : Annual direct costs per treated patient

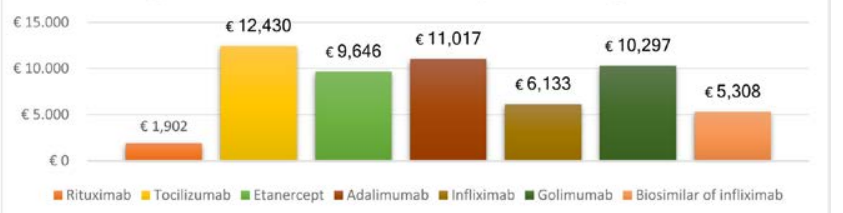

Conclusion: This study presents a global overview of the direct costs of biological therapy associated with RA patients, taking as a source the Moroccan registry of biological therapies in rheumatic diseases. Those results show that overall cost of biological agents according to the Moroccan register is very high. There is a real need for development of strategies for better governance of those costs. Disclosure of Interests: None declared

DOI: 10.1136/annrheumdis-2021-eular.4071

\section{Epidemiology, risk factors for disease or disease progression}

\begin{tabular}{|l|l|}
\hline AB0812 & BIOMARKERS FOR CONNECTIVE TISSUE DISEASE- \\
ASSOCIATED INTERSTITIAL LUNG DISEASE: A PILOT \\
STUDY
\end{tabular}

Y. H. Chiu ${ }^{1,2}$, M. Voortman ${ }^{3}$, E. M. Delemarre ${ }^{4}$, S. Nierkens ${ }^{4}$, P. De Jong ${ }^{5}$, F. Mohamed Hoesein ${ }^{5}$, J. M. Van Laar ${ }^{1}$, J. Spierings ${ }^{1} .{ }^{1}$ University Medical
Centre Utrecht, Department of Rheumatology and Clinical Immunology, Utrecht, Netherlands; ${ }^{2}$ National Defense Medical Center, Division of Rheumatology/ Immunology/Allergy, Taipei, Taiwan, Republic of China; ${ }^{3}$ University Medical Centre Utrecht, Department of Heart and Lung, Utrecht, Netherlands;

${ }^{4}$ University Medical Centre Utrecht, Center for Translational Immunology, Utrecht, Netherlands; ${ }^{5}$ University Medical Centre Utrecht, Department of Radiology, Utrecht, Netherlands

Background: Connective tissue disease (CTD)-associated interstitial lung disease (ILD) has high morbidity and increased mortality risk. Biomarkers that can predict prognosis and response to therapy have been studied in recent years, including cytokines, chemokines, adhesion molecules, growth factors, extracellular remodelling molecules and pneumocyte associated proteins. However, there is still a knowledge gap for interpreting biomarkers in the context of imaging and response.

Objectives: The aim of our study was to identify biomarkers that are associated with inflammatory and fibrotic changes over time on high-resolution computed tomography (HRCT) and response to treatment in CTD-ILD.

Methods: An exploratory set of 38 biomarkers were measured in serum of patients with CTD-ILD using a multiplex immunoassay, chemiluminescence enzyme immunoassay and enzyme-linked immunosorbent assay Samples were taken at baseline and one year of follow-up. Clinical data was extracted from electronic medical patients records. Two experienced chest radiologists independently and blindly reviewed the HRCTs. A third expert reviewed the scan in case of discrepancies. They classified ILD patterns according to the classification for idiopathic interstitial pneumonia and categorised into fibrotic or inflammatory. Association between biomarkers at baseline and pulmonary function test (PFT) and HRCT changes in response to treatment were analysed by logistic regression. Responsiveness of biomarkers to treatment was determined using the paired Wilcoxon sign rank test. Correlations between the variation of biomarkers and PFT after one year of treatment were examined using Spearman's rank correlation coefficient.

Results: Sixteen patients were included (12 females $(75.0 \%)$, median age 51 years (IQR 45-62). Underlying CTDs were systemic sclerosis $(n=5)$, Sjogren's syndrome $(n=1)$, inflammatory myopathies $(n=6)$, rheumatoid arthritis $(n=1)$, systemic lupus erythematosus $(n=1)$, mixed connective tissue disease $(n=1)$, and undifferentiated connective tissue disease $(n=1)$. There were three patients with a fibrotic HRCT pattern and 13 patients with an inflammatory HRCT pattern.

Biomarkers attenuation in CXCL11, CTGF, and KL-6 were associated with inflammatory HRCT patterns as well as less HRCT progression and fewer FVC decline. Furthermore, a decrease in CTGF was observed in patients treated with MMF and corticoids, while a reduction in CXCL11 and SPD were seen in patients treated with hydroxychloroquine. (Table 1) Additionally, an increase in levels of galectin-3 at one-year follow-up was associated with improved predicted FVC (Rho 0.5, p=0.048). (Figure 1)

Table 1. Biomarker responses to clinical treatment

\begin{tabular}{lccccc}
\hline Treatment & Biomarkers & $\begin{array}{c}\text { Baseline, Median } \\
(\text { IQR })\end{array}$ & $\begin{array}{c}\text { Follow up, } \\
\text { Median (IQR) }\end{array}$ & $\begin{array}{c}\text { Variation } \\
\text { percentage }\end{array}$ & $p$ \\
\hline MMF & CTGF $(\mathrm{pg} / \mathrm{ml})$ & $\begin{array}{c}110.69 \\
(63.21-158.06)\end{array}$ & $4.73(4.73-45.3)$ & -95.73 & 0.036 \\
& & & & \\
$(\mathrm{n}=7)$ & sVCAM $(\mathrm{pg} / \mathrm{ml})$ & 2061300 & 1767600 & -14.25 & 0.031 \\
& & $(1939200-$ & $(1707100-$ & & \\
& & $3042250)$ & $1986350)$ & & \\
HCQ & CXCL11 $(\mathrm{pg} / \mathrm{ml})$ & 699.30 & 333.39 & -52.33 & 0.031 \\
& & $(392.50-764.50)$ & $(223.1-487.92)$ & & \\
$(\mathrm{n}=6)$ & SPD $(\mathrm{ng} / \mathrm{ml})$ & 18.70 & 16.80 & -10.16 & 0.031 \\
& & $(13.40-28.73)$ & $(11.70-22.80)$ & & \\
Steroid & CTGF $(\mathrm{pg} / \mathrm{ml})$ & 81.93 & 12.76 & -84.43 & 0.044 \\
& & $(20.80-158.06)$ & $(4.73-56.74)$ & & \\
$(\mathrm{n}=11)$ & MMP-7 $(\mathrm{pg} / \mathrm{ml})$ & 3146.3 & 4563.3 & 45.04 & 0.005 \\
& & $(1779.7-3997.0)$ & $(2951.9-7625.8)$ & & \\
\hline
\end{tabular}

IQR, interquartile range; MMF, mycophenolate mofetil; $\mathrm{HCQ}$, hydroxychloroquine; CTGF, connective tissue growth factor; sVCAM, soluble vascular cell adhesion molecule-1; SPD, surfactant protein D; MMP-7, matrix metalloproteinase 7.

Conclusion: In this pilot study, CXCL11, CTGF, and KL-6 reduction were associated with inflammatory HRCT patterns and better pulmonary outcome. In contrast to previous research in severe ILD, there was a positive correlation between changes of Galectin-3 and FVC in our study. Further research in a larger group and focusing on combining biomarkers to predict outcome and prognosis are needed. 